\title{
Baicalein pretreatment confers cardioprotection against acute myocardial infarction by activating the endothelial nitric oxide synthase signaling pathway and inhibiting oxidative stress
}

\author{
SHENGYU TAN $^{1}$, SHENGHUA ZHOU ${ }^{2}$ and YINGQUAN LUO ${ }^{1}$ \\ Departments of ${ }^{1}$ Geriatrics and ${ }^{2}$ Cardiology, Second Xiangya Hospital, Central South University, \\ Changsha, Hunan 410011, P.R. China
}

Received August 2, 2013; Accepted March 3, 2014

DOI: $10.3892 / \mathrm{mmr} .2014 .2091$

\begin{abstract}
Baicalein, a flavonoid purified from the root of Scutellaria baicalensis Georgi, is well known for its anti-inflammatory and anti-oxidative properties. The present study aimed to evaluate the cardioprotective effect of baicalein in a rat model of acute myocardial infarction and investigate the potential molecular mechanisms of this effect. The acute myocardial infarction model was prepared by permanently occluding the left anterior descending artery. Baicalein and/or the endothelial nitric oxide synthase (eNOS) inhibitor L-NAME was injected prior to the induction of acute myocardial infarction. In the vehicle-treated group, acute myocardial infarction resulted in a markedly increased infarction size and elevated levels of plasma cardiac enzymes, including creatine kinase, the MB isoenzyme of creatine kinase, lactate dehydrogenase and cardiac troponin $\mathrm{T}$, compared with those in the sham-surgery group. In the baicalein treatment group, the infarcted area and plasma levels of the cardiac enzymes were significantly decreased compared with those in the vehicle-treated group. In addition, pretreatment with baicalein potently increased the levels of eNOS protein and nitric oxide production in the infarcted rats. Furthermore, myocardial oxidative stress was attenuated by baicalein preconditioning following acute myocardial infarction. However, L-NAME inihibted the cardioprotective effects of baicalein. These data indicate that baicalein protects against acute myocardial infarction-induced injury by activating eNOS signaling and inhibiting oxidative stress.
\end{abstract}

Correspondence to: Dr Yingquan Luo, Department of Geriatrics, Second Xiangya Hospital, Central South University, 139 Renmin Road, Changsha, Hunan 410011, P.R. China

E-mail: luoshazhaomr@163.com

Key words: baicalein, acute myocardial infarction, cardioprotection, endothelial nitric oxide, oxidative stress

\section{Introduction}

Nitric oxide (NO) is a fundamental endothelium-derived relaxing factor involved in a variety of physiological processes within organ systems. Under physiological conditions, NO has the characteristic of inhibiting leukocyte and smooth muscle cell hyperplasia, and is also beneficial in preventing cardiac damage following myocardial ischemia (1). Emerging evidence has indicated that NO exerts cardioprotection during ischemia-reperfusion events $(2,3)$. NO is catalyzed from the substrate L-arginine by nitric oxide synthase (NOS). At least three distinct subgroups, including neuronal NOS (nNOS or NOS1), inducible NOS (iNOS or NOS2) and endothelial cell NOS (eNOS or NOS3), are classified into the NOS family in mammalian cells. Among these isoforms, eNOS is highly expressed in coronary endothelial cells and is also rich in the sarcolemma of cardiac myocytes (4). NO released from eNOS in the heart is considered to have a critical role in coronary vasodilation and tonic inhibition of mitochondrial $\mathrm{O}_{2}$ consumption. A study demonstrated that transgenic mice overexpressing eNOS were protected from myocardial infarction-induced cardiac damage (5). Conversely, progressive cardiomyocyte dysfunction was evidently exacerbated in eNOS $^{-/}$mice following myocardial infarction, suggesting a protective effect of eNOS-derived NO $(6,7)$. Therefore, inhibition of eNOS may be a useful and promising approach for attenuating the impairment caused by myocardial infarction.

Cumulative evidence has also demonstrated that oxidative stress is implicated in the pathogenesis of myocardial infarction, and finally contributes to cardiac dysfunction and the death of cardiomyocytes $(8,9)$. A study identified that marked generation of oxygen-derived free radicals following ischemic insults, which destroyed endogenous antioxidant defenses and resulted in oxidative damage of membrane lipids, proteins, carbohydrates and DNA, concomitantly leading to cardiomyocyte necrosis and apoptosis (10). A previous study revealed that excessive free radicals attack the unsaturated fatty acids within myocardial membranes and exacerbate the damage following acute myocardial infarction (11).

Baicalein is a medicinal herb purified from the root of Scutellaria baicalensis Georgi and has been demonstrated to possess anti-inflammatory (12) and anti-oxidative (13) 
properties. A previous study revealed that baicalein protected cardiomyocytes against ischemia-reperfusion injury in a chick embryonic ventricular myocyte model (14). In addition, Woo et al (15) also demonstrated that pretreatment with baicalein evidently diminished intracellular calcium overload and attenuated the cardiac damage during myocardial ischemia-reperfusion injury. However, whether baicalein has is protective against myocardial infarction in rats has not been clarified. Furthermore, as the eNOS signaling pathway and oxidative stress are central in the amelioration of ischemic insults, we hypothesized that they are involved in the cardioprotective effect of baicalein. The present study was performed to evaluate the cardiprotective effect of baicalein and elucidate the roles of the eNOS signaling pathway and oxidative stress in baicalein-mediated cardioprotection against acute myocardial infarction in rats.

\section{Materials and methods}

Experimental animals. All experiments were conducted with adult male Wistar rats (weight, 230-260 g), obtained from the Beijing Animal Center (Beijing, China) and allowed to acclimatize in the animal cage for four days prior to use, with access to water and food ad libitum. All experimental protocols were approved by the Animal Care Committee of the Second Xiangya Hospital of Central South University, (Changsha, Hunan, China). Efforts were made to minimize suffering and the number of animals used.

Acute myocardial infarction model and drug administration. The induction of acute myocardial infarction was performed as previously described (16). In brief, the male Wistar rats were anesthetized with pentobarbital sodium (40 mg/kg) intraperitoneally (i.p.) and then fixed on the operating table for the surgical procedures. A tracheotomy was performed and an intubation cannula was connected with a volume-controlled ventilator (Nanjing Jiancheng Biotechnology Institute, Nanjing, Jiangsu, China). The normal electrocardiogram (II; Nanjing Jiancheng Biotechnology Institute) was recorded via a multi-channel recorder (BL-420F; Chengdu Taimeng, Chengdu, China) after the electrodes were subcutaneously placed onto the four limbs and connected to an electrocardiograph. A 5-0 silk suture 1-2 mm (Nanjing Jiancheng Biotechnology Institute) was employed to encircle the left anterior descending coronary artery below the left atrial appendage. The left coronary artery of the rats was ligated. The sham-surgery animals underwent identical surgical procedures with the exception of coronary artery occlusion. Baicalein (purity $>95 \%$; Sigma-Aldrich, St. Louis, MO, USA) was dissolved in dimethylsulfoxide (DMSO; $0.01 \%$; diluted in phosphate-buffered saline). The rats were randomized into five groups as follows: i) Sham-surgery group (Sham; $n=6$ ), rats underwent identical surgery, with the exception of coronary artery ligation, and were administered with $0.01 \%$ DMSO (0.1 ml/100 g, i.p.); ii) vehicle group (Vehicle; $\mathrm{n}=6$ ), rats underwent occlusion of the left coronary artery and were administered with $0.01 \%$ DMSO $(0.1 \mathrm{ml} / 100 \mathrm{~g}$, i.p.); iii) baicalein treatment group (BAC $30 ; n=6)$, rats were subjected to occlusion of the left coronary artery and treated with baicalein (30 mg/kg, i.p.) (17); iv) L-NAME (an inhibitor of NOS) treatment group (L-NAME; n=6), rats were subjected to occlusion of the left coronary artery and treated with L-NAME (10 mg/kg, i.p.) (18); v) baicalein and L-NAME co-administration group (BAC30 + L-NAME; $n=6)$, rats were subjected to occlusion of the left coronary artery and treated with baicalein (30 $\mathrm{mg} / \mathrm{kg}$, i.p.) and L-NAME (10 mg/kg, i.p.). Baicalein and DMSO were administered once a day for six consecutive days and L-NAME was injected once $25 \mathrm{~min}$ prior to the induction of ischemia. After the final administration (30 min), the rats underwent surgery by occlusion of the left coronary artery.

Measurement of infarct size. After the occlusion of the coronary artery $(6 \mathrm{~h})$, the animals were anesthetized with pentobarbital sodium and the hearts were immediately excised, and the left ventricles were then sectioned into transverse slices (2-mm thick) from the apex to the atrioventricular groove. The slices were subsequently incubated in $1 \%$ triphenyltetrazolium chloride (TTC; Sigma-Aldrich) solution at $37^{\circ} \mathrm{C}$ for $30 \mathrm{~min}$, photographed with a digital camera (Panasonic Lumix DMC-TZ40-K; Canon Inc., Tokyo, Japan) and weighed. For each slice, TTC stained the normal myocardium brick-red while the infarcted area remained grayish-white. The size of the infarcted area was calculated by the volume and weight as a percentage of the left ventricle, according to the method described in a previous study (16).

Determination of plasma cardiac marker enzyme levels. The cardiac damage was evaluated by measuring the levels of myocardial-specific enzymes, including creatine kinase (CK), MB isoenzyme of creatine kinase (CK-MB), lactate dehydrogenase (LDH) and cardiac troponin T (cTnT). The blood samples were acquired $6 \mathrm{~h}$ after the occlusion of the coronary artery. The plasma concentrations of CK, CK-MB and LDH were measured by the colorimetric method using an automatic biochemical analyzer (AU-2700; Olympus Corporation, Tokyo, Japan). The plasma cTnT levels were measured by an immunoassay kit (Elecsys 2010; Roche Diagnostics, Mannheim, Germany).

Western blotting. The heart samples were collected $6 \mathrm{~h}$ after the occlusion of the coronary artery and homogenized in a standard lysis buffer. Following centrifugation at $13,200 \times \mathrm{g}$ for $20 \mathrm{~min}$ at $4^{\circ} \mathrm{C}$, the supernatant was collected and the protein concentration was determined by a bicinchoninic acid assay (Beyotime Institute of Biotechnology, Shanghai, China). Protein (30 $\mu \mathrm{g})$ was separated on $8 \%$ sodium dodecyl sulfate-polyacrylamide gel electrophoresis gels and transferred to nitrocellulose membranes (Nanjing Jiancheng Biotechnology Institute). The protein was detected using rabbit anti-eNOS (1:1,000; Sigma-Aldrich) or mouse anti-GAPDH (1:2,000; KangChen Bio-tech, Shanghai, China) and horseradish peroxidase-conjugated goat anti-rabbit antibody (1:5,000; Santa Cruz Biotechnology, Inc., Santa Cruz, CA, USA) or goat anti-mouse antibody (1:5,000; Santa Cruz Biotechnology, Inc.), respectively. Detection of the labeled antigens was performed with an enhanced chemiluminescence kit (Nanjing Jiancheng Biotechnology Institute). Densitometric analysis was conducted using Quantity One 1-D analysis software (Bio-Rad, Hercules, CA, USA). 
Assay of plasma NO production levels. The blood samples were obtained $6 \mathrm{~h}$ after the occlusion of the coronary artery and centrifuged at $2,000 \mathrm{x}$ g for $20 \mathrm{~min}$ at $4{ }^{\circ} \mathrm{C}$. The plasma supernatant was collected and the nitrite concentration was spectrophotometrically determined using Griess reagent (Nanjing Jiancheng Biotechnology Institute; 1\% sulfanilamide and $0.1 \%$ naphthylethylenediamide in $5 \%$ phosphoric acid). The absorbance was measured at $540 \mathrm{~nm}$ and the nitrite concentration was calculated using sodium nitrite as a standard.

Measurement of malondialdehyde (MDA) and superoxide dismutase (SOD) levels. The myocardial supernatant was isolated by centrifugation, at $3,000 \mathrm{x}$ g for $10 \mathrm{~min}$ at $4^{\circ} \mathrm{C}, 6 \mathrm{~h}$ after the occlusion of the coronary artery. The MDA levels in the supernatant were determined by the measurement of thiobarbituric-acid reacting substances with a commercial kit (Nanjing Jiancheng Bioengineering Institute, Nanjing, China) according to the manufacturer's instructions. The SOD activity levels in the supernatant were estimated in the heart homogenate by calculating the rate of the inhibition of nucleotide oxidation using a Superoxide Dismutase Detection kit (A001-3; Nanjing Jiancheng Bioengineering Institute).

Detection of plasma 8-isoprostane levels. The levels of 8-isoprostane in each group were analyzed using an 8-Isoprostane EIA kit (no. 516351; Cayman Chemical Company, Ann Arbor, MI, USA) according to the manufacturer's instructions. Briefly, the plasma samples were hydrolyzed by adding $25 \mu \mathrm{l}$ of $2 \mathrm{M} \mathrm{NaOH}$ to each $100 \mu \mathrm{l}$ plasma sample and the samples were then incubated at $45^{\circ} \mathrm{C}$ for $2 \mathrm{~h}$. Following the addition of $25 \mu \mathrm{l}$ of $10 \mathrm{M} \mathrm{HCl}$ acid, the samples were centrifuged at 12,000 x $\mathrm{g}$ for $5 \mathrm{~min}$. The supernatant was collected and the measurement of 8-isoprostane was performed using the commercial kit. The results were expressed as $\mathrm{pg} / \mathrm{ml}$ of plasma.

Statistical analysis. Data were collected and are presented as the mean \pm standard deviation. Comparisons between different groups were performed using one-way analysis of variance (ANOVA) followed by Dunnett's test. All statistical analyses were conducted using SPSS software, version 13.0 (SPSS, Inc., Chicago, IL, USA). $\mathrm{P}<0.05$ was considered to indicate a statistically significant difference.

\section{Results}

Baicalein reduces myocardial infarct size in a rat model of acute myocardial infarction. The chemical structure of baicalein is shown in Fig. 1. As shown in Fig. 2, the infarction size in the vehicle-treated infarcted group was $41.96 \pm 3.20 \%$. However, the baicalein pretreatment yielded an infarct size of $28.36 \pm 3.75 \%(\mathrm{P}<0.01$, versus the vehicle group). Notably, when baicalein and L-NAME, an inhibitor of NOS, were co-administered, the infarcted area was significantly increased to $38.59 \pm 3.39 \%(\mathrm{P}<0.01)$, compared with that of the baicalein-treated group.

Baicalein inhibits plasma $C K, C K-M B$ and $L D H$ activity and reduces cTnT levels in a rat model of acute myocardial infarction. To investigate whether baicalein inhibited the

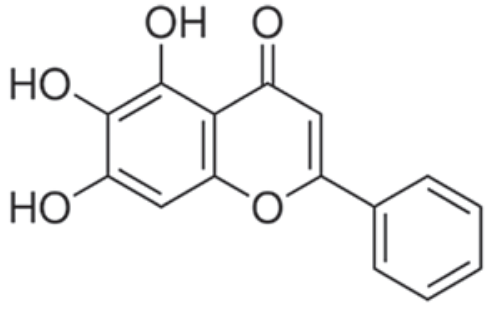

Figure 1. Chemical structure of baicalein.

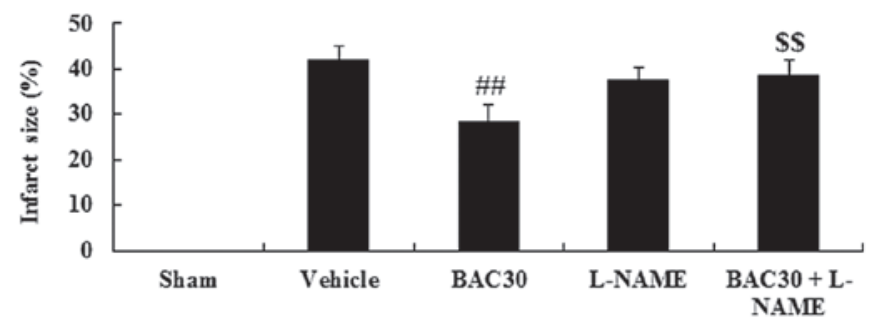

Figure 2. Baicalein reduces the myocardial infarct size in a rat model of acute myocardial infarction (mean \pm standard deviation; $n=6$ ). ${ }^{\# \#} \mathrm{P}<0.01$ vs. the vehicle-treated group; ${ }^{\$ \$} \mathrm{P}<0.01$ vs. the baicalein-treated group. Sham, sham-surgery; vehicle, vehicle-treated; BAC30, baicalein $(30 \mathrm{mg} / \mathrm{kg})$-treated; L-NAME, L-NAME (10 mg/kg)-treated; and BAC30 + L-NAME, co-administration of baicalein $(30 \mathrm{mg} / \mathrm{kg})$ and L-NAME $(10 \mathrm{mg} / \mathrm{kg})$-treated groups.

cardiomyocyte necrosis, the activity of plasma CK, CK-MB and LDH, as well as the cTnT levels were determined at the end of the surgery. As displayed in Fig. 3, the levels of CK, CK-MB, LDH and cTnT in the vehicle-treated group were markedly elevated $(\mathrm{P}<0.01)$, in comparison with those in the sham group. Following preconditioning with baicalein, the specific cardiac enzymes were all significantly decreased in the plasma compared with those in the vehicle controls $(\mathrm{P}<0.01)$. Furthermore, pretreatment with L-NAME completely inhibited the baicalein-induced reduction in the levels of $\mathrm{CK}$, CK-MB, LDH and cTnT in the plasma of infarcted rats versus those in the baicalein treatment group $(\mathrm{P}<0.01)$.

Baicalein increases eNOS protein expression levels and plasma NO concentration in a rat model of acute myocardial infarction. Whether baicalein exerted protection against acute myocardial infarction through mediating the eNOS signaling pathway was further investigated. Fig. 4A reveals that western blotting with the eNOS antibody produced specific bands of $133 \mathrm{kDa}$. Following the one-way ANOVA, an evident reduction in the levels of eNOS protein was observed in the vehicle-treated group $(\mathrm{P}<0.01)$, versus those of the sham control, as shown in Fig. 4B. However, baicalein pretreatment caused a significant elevation in the eNOS protein levels in the myocardial infarction-induced rats $(\mathrm{P}<0.01)$, compared with those in the vehicle group. Co-administration of baicalein and L-NAME completely inhibited the increase in eNOS protein expression levels caused by baicalein $(\mathrm{P}<0.01, \mathrm{n}=6)$. In addition, the levels of NO production were also evaluated. The levels of NO production, which were indicated as the levels of nitrite formation, were reported to be significantly reduced following the induction of acute myocardial infarction $(\mathrm{P}<0.01)$. Baicalein pretreatment induced a marked increase in 

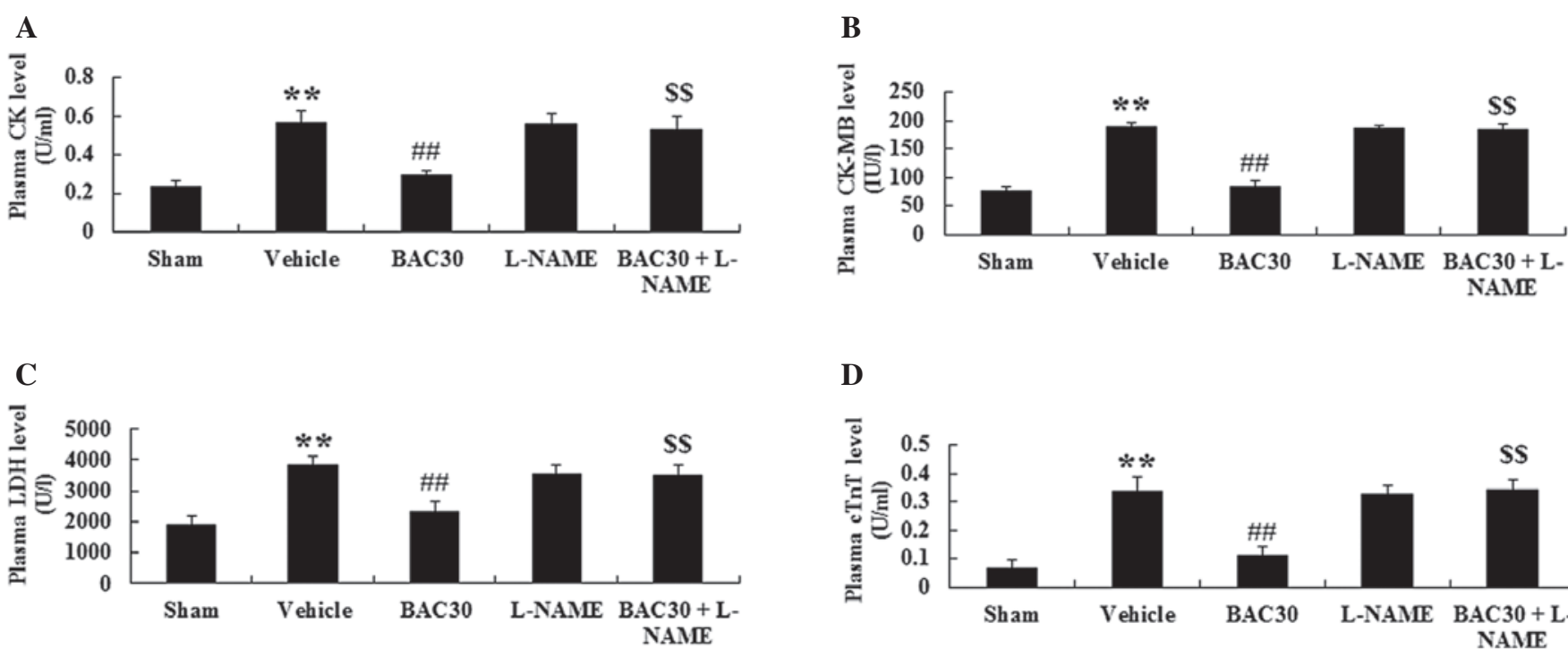

D

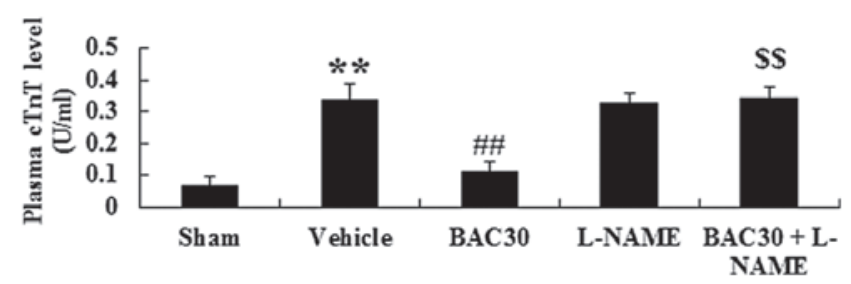

Figure 3. Baicalein inhibits plasma CK, CK-MB and LDH activity and reduces cTnT levels in a rat model of acute myocardial infarction (mean \pm standard deviation; $\mathrm{n}=6$ ). The plasma (A) CK, (B) CK-MB and (C) LDH activity and (D) cTnT levels in the different groups. ${ }^{* *} \mathrm{P}<0.01$ vs. the sham-surgery group; ${ }^{\# \#} \mathrm{P}<0.01$ vs. the vehicle-treated group and ${ }^{\$ \$} \mathrm{P}<0.01$ vs. the baicalein-treated group. Sham, sham-surgery; vehicle, vehicle-treated; BAC30, baicalein (30 mg/kg)-treated; L-NAME, L-NAME $(10 \mathrm{mg} / \mathrm{kg})$-treated; and BAC30 + L-NAME, co-administration of baicalein $(30 \mathrm{mg} / \mathrm{kg})$ and L-NAME (10 mg $/ \mathrm{kg})$-treated groups. CK, creatine kinase; CK-MB, MB isoenzyme of creatine kinase; $\mathrm{LDH}$, lactate dehydrogenase; cTnT, cardiac troponin T.

A
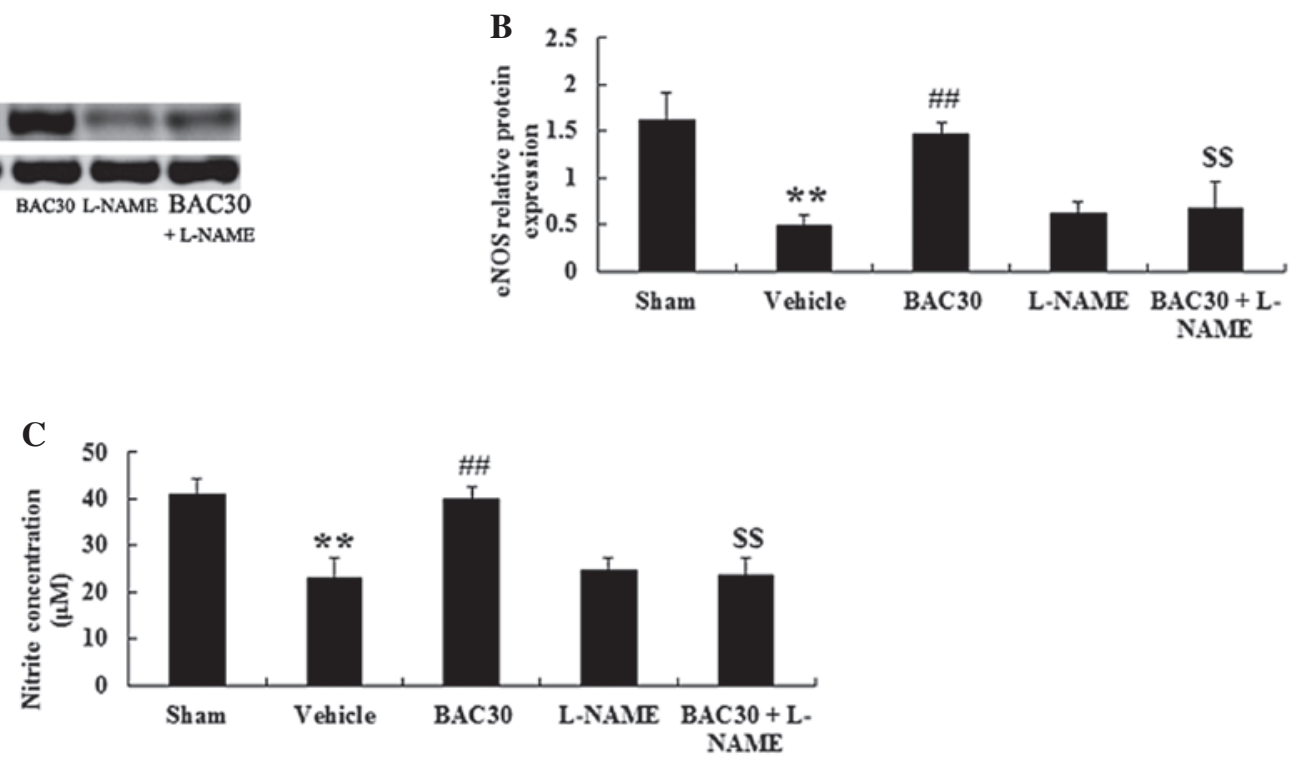

Figure 4. Baicalein increases the eNOS protein expression levels and plasma NO concentration in a rat model of acute myocardial infarction (mean \pm standard deviation; $\mathrm{n}=6$ ). (A) Representative images of the immunoblots with antibodies against eNOS in hearts of the rats from different groups. eNOS, $133 \mathrm{kDa}$ and GAPDH, $36 \mathrm{kDa}$. (B) Quantitative analysis of the protein levels of eNOS in hearts of the rats from different groups. The data were normalized to the loading control GAPDH. (C) NO production was detected spectrophotometrically by measuring the levels of its metabolite, nitrite. ${ }^{* *} \mathrm{P}<0.01 \mathrm{vs}$. the sham-surgery group; ${ }^{\# \#} \mathrm{P}<0.01$ vs. the vehicle-treated group; ${ }^{\$ \$} \mathrm{P}<0.01$ vs. the baicalein-treated group. Sham, sham-surgery; vehicle, vehicle-treated; BAC30, baicalein $(30 \mathrm{mg} / \mathrm{kg})$-treated; L-NAME, L-NAME $(10 \mathrm{mg} / \mathrm{kg})$-treated; and BAC30 + L-NAME, co-administration of baicalein $(30 \mathrm{mg} / \mathrm{kg})$ and L-NAME $(10 \mathrm{mg} / \mathrm{kg})$-treated groups. eNOS, endothelial nitric oxide synthase; NO, nitric oxide.

the nitrite levels $(\mathrm{P}<0.01)$ compared with those in the vehicle group; however, this elevation was suppressed in the presence of L-NAME (P<0.01; Fig. 4C).

Baicalein inhibits oxidative stress in a rat model of acute myocardial infarction via eNOS signaling. To examine the effect of baicalein on oxidative stress during acute myocardial infarction, the MDA and SOD levels were detected in the myocardium of different groups. Compared with those of the sham group, the MDA content was markedly increased while the SOD activity levels were reduced $(\mathrm{P}<0.01)$. Following preconditioning with baicalein, the MDA levels were markedly reduced $(\mathrm{P}<0.01)$ compared with those of the vehicle-treated group. However, the reduced effect of MDA in the baicalein treatment group was reversed by the administration of L-NAME (Fig. 5A). By contrast, baicalein pretreatment in the infarcted rats induced an evident increase in the levels of SOD activity compared with those in the vehicle group and this phenomenon 
A

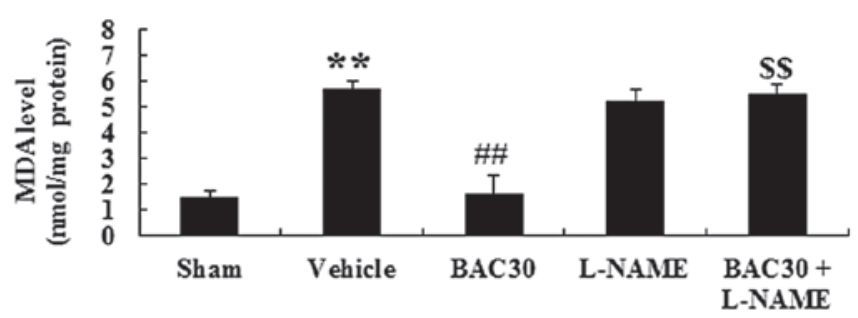

B
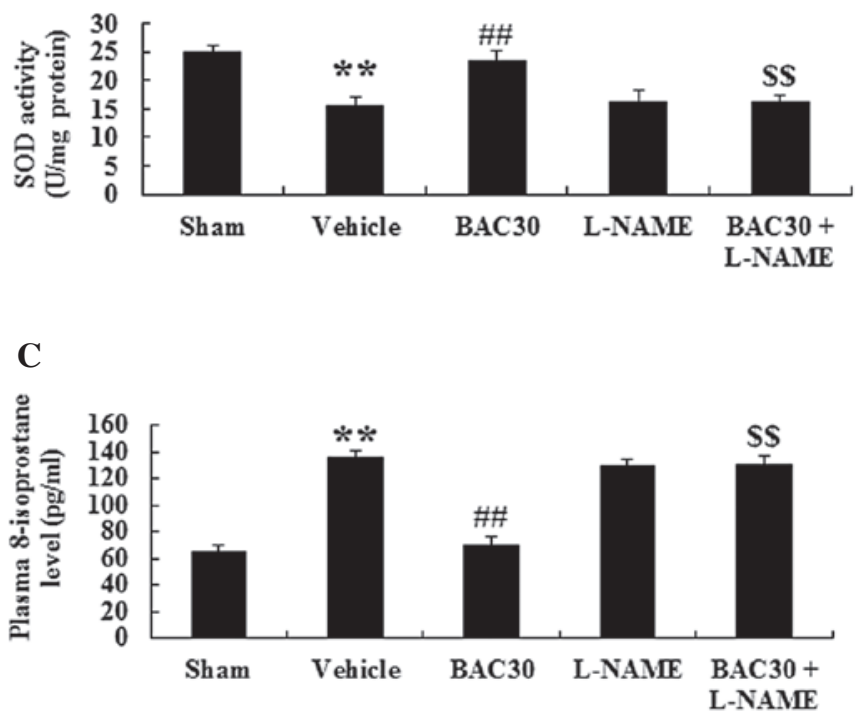

Figure 5. Baicalein inhibits the oxidative stress in a rat model of acute myocardial infarction via eNOS signaling (mean \pm standard deviation; $n=6$ ). The levels of (A) MDA, (B) SOD and (C) 8-isoprostane in the different groups. ${ }^{* *} \mathrm{P}<0.01$ vs. the sham-surgery group; ${ }^{\# \#} \mathrm{P}<0.01$ vs. the vehicle-treated group ${ }^{\$ \$} \mathrm{P}<0.01$ vs. the baicalein-treated group. Sham, sham-surgery; vehicle, vehicle-treated; BAC30, baicalein $(30 \mathrm{mg} / \mathrm{kg}$ )-treated; L-NAME, L-NAME $(10 \mathrm{mg} / \mathrm{kg})$-treated; and BAC30 + L-NAME, co-administration of baicalein $(30 \mathrm{mg} / \mathrm{kg})$ and L-NAME $(10 \mathrm{mg} / \mathrm{kg})$-treated groups. MDA, malondialdehyde; SOD, superoxide dismutase.

was inhibited by L-NAME (Fig. 5B). The levels of 8-isoprostane, an important index for evaluating oxidative damage (19), were also determined. As shown in Fig. 5C, acute myocardial infarction resulted in a marked augmentation in the levels of 8 -isoprostane compared with those in the sham group $(\mathrm{P}<0.01$, $\mathrm{n}=6$ ). Baicalein treatment at the dose of $30 \mathrm{mg} / \mathrm{kg}$ significantly reduced the concentration of 8 -isoprostane $(\mathrm{P}<0.01)$ compared with that of the vehicle group. However, the suppression of eNOS signaling by the inhibitor L-NAME completely abolished the baicalein-induced reduction of 8-isoprostane content $(\mathrm{P}<0.01)$. Collectively, baicalein pretreatment markedly attenuated the oxidative stress following acute myocardial infarction and this attenuation may be associated with eNOS signaling.

\section{Discussion}

The major findings in the present study demonstrated that pretreatment with baicalein had the capacity to reduce infarct size and the activity of specific cardiac enzymes (CK, CK-MB, LDH and cTnT). The protective effects induced by baicalein preconditioning may be associated with activation of eNOS and an increase in NO bioavailability, together with inhibition of oxidative stress. These protective effects were also abolished by an eNOS inhibitor (L-NAME), suggesting that an eNOS-dependent mechanism was involved in the baicalein-mediated cardioprotection.

In the rat model of acute myocardial infarction in the present study, notably the baicalein treatment markedly diminished the infarction size and the levels of cardiac marker enzymes (CK, CK-MB, LDH and cTnT). During ischemic insults, these myocardial enzymes and protein are released into the blood and their concentrations in the plasma directly reflect the extent of the myocardial injury. Therefore, CK, CK-MB, LDH and cTnT in the plasma are sensitive biomarkers for assessing cardiac injury (11). Previous studies have shown that the infarction size and the levels of myocardial specific enzymes, including CK, CK-MB and LDH, were all markedly elevated in rats subjected to acute myocardial infarction $(20,21)$. cTnT is a contractile protein, which remains at low levels but is markedly augmented during myocardial necrosis or cell death (22). In line with the results of previous studies, the present study demonstrated elevations in infarct size and the levels of myocardial specific enzymes (CK, CK-MB, LDH and cTnT) in the infarcted rats compared with those in the sham group. However, they were all markedly decreased following baicalein administration, suggesting the cardioprotective effect of baicalein against acute myocardial infarction. Notably, the attenuation of cardiac damage was completely inhibited following co-treatment with baicalein and an eNOS inhibitor (L-NAME). This implies that baicalein prevents cardiomyocytes from injury during acute myocardial infarction, at least in part, via mediating eNOS signaling.

$\mathrm{NO}$ is regarded as one of the major regulators during myocardial damage. A prior study revealed that eNOS-derived $\mathrm{NO}$ exerts protection against myocardium injury caused by myocardial ischemia-reperfusion (7). In addition, it has been demonstrated that treatment with statins significantly attenuates the impairment of ischemia-reperfusion during myocardial infarction, via eNOS-mediated signaling pathways with increased NO production (23). Consistent with the results of previous studies, the present study demonstrated that the levels of protein expression and NO production were evidently reduced in the infarcted rats. Administration of baicalein markedly augmented the protein levels of eNOS and promoted NO generation in the myocardial infarction model. More importantly, baicalein-induced increases in the levels of eNOS and NO production in the infarcted rats were completely inhibited by the presence of an eNOS inhibitor (L-NAME). These findings suggest that baicalein exerted protective effects against acute myocardial infarction by activating eNOS and concomitantly increasing NO bioavailability.

Oxidative stress has also been implicated in the pathogenesis of cardiac dysfunction and the death of cardiomyocytes. Excessively produced oxygen free radicals attack the fatty acids within the myocardial membranes and cause the release of lipid peroxides (11). Accumulation of lipid hydroperoxides directly reflects the damage of the cardiac constituents. MDA is a major lipid peroxidation end product and an increased MDA concentration has been demonstrated to facilitate the generation of free radicals and inhibit the activity of the antioxidant defense system (24). SOD is conceived of as the first line of cell defense against oxidative stress, which functions 
by eliminating reactive oxygen radicals, including superoxide and hydrogen peroxide, and preventing the generation of more hydroxyl radicals. In the present study, acute myocardial infarction resulted in a marked elevation in the MDA content and reduced the SOD activity levels compared with those in the sham group, indicating the generation of oxidative stress. The levels of 8 -isoprostane, a cardiac marker of oxidative stress, were also identified as evidently increased in the infarcted rats compared with those in the sham group and this finding confirmed the production of oxidative stress during acute myocardial infarction. Baicalein pretreatment induced significant reductions in the levels of MDA and 8-isoprostane, while increasing those of SOD activity, which is attributable to its potent anti-oxidative property. In agreement with the findings of the present study, a previous study reported that baicalein preconditioning protected cardiomyocytes from oxidative damage in a chick embryonic model of ischemia-reperfusion injury in vitro (25). Furthermore, the present study also identified that inhibition of eNOS by L-NAME significantly prevented the baicalein-mediated protection against oxidative damage, implying the involvement of eNOS signaling in the attenuation of oxidative stress by baicalein pretreatment following acute myocardial infarction.

In conclusion, to the best of our knowledge, the present study demonstrated for the first time that baicalein, a natural occurring flavonoid, has potent cardioprotective effects in rats with acute myocardial infarction, and that the cardioprotective effects may be associated with activation of eNOS signaling and inhibition of oxidative stress. Furthermore, the inhibition of oxidative stress by baicalein treatment is eNOS-dependent. These findings support the use of baicalein as a promising cardioprotective agent for the prevention or treatment of myocardial injury induced by acute myocardial infarction. This study also suggests that eNOS signaling may serve as a potential therapeutic target in ischemic heart disease in the future.

\section{References}

1. Bulhak AA, Sjöquist PO, Xu CB, Edvinsson L and Pernow J: Protection against myocardial ischaemia/reperfusion injury by PPAR-alpha activation is related to production of nitric oxide and endothelin-1. Basic Res Cardiol 101: 244-252, 2006.

2. Gourine AV, Bulhak AA, Gonon AT, Pernow J and Sjöquist PO: Cardioprotective effect induced by brief exposure to nitric oxide before myocardial ischemia-reperfusion in vivo. Nitric Oxide 7: 210-216, 2002.

3. Andelová E, Barteková M, Pancza D, Styk J and Ravingerová T: The role of NO in ischemia/reperfusion injury in isolated rat heart. Gen Physiol Biophys 24: 411-426, 2005.

4. Balligand JL and Cannon PJ: Nitric oxide synthases and cardiac muscle. Autocrine and paracrine influences. Arterioscler Thromb Vasc Biol 17: 1846-1858, 1997.

5. Jones SP, Greer JJ, van Haperen R, Duncker DJ, de Crom R and Lefer DJ: Endothelial nitric oxide synthase overexpression attenuates congestive heart failure in mice. Proc Natl Acad Sci USA 100: 4891-4896, 2003.

6. Sumeray MS, Rees DD and Yellon DM: Infarct size and nitric oxide synthase in murine myocardium. J Mol Cell Cardiol 32: $35-42,2000$.
7. Jones SP, Girod WG, Palazzo AJ, et al: Myocardial ischemia-reperfusion injury is exacerbated in absence of endothelial cell nitric oxide synthase. Am J Physiol 276: H1567-H1573, 1999

8. Carden DL and Granger DN: Pathophysiology of ischaemia-reperfusion injury. J Pathol 190: 255-266, 2000.

9. Collard CD and Gelman S: Pathophysiology, clinical manifestations, and prevention of ischemia-reperfusion injury. Anesthesiology 94: 1133-1138, 2001.

10. Panda VS and Naik SR: Cardioprotective activity of Ginkgo biloba Phytosomes in isoproterenol-induced myocardial necrosis in rats: a biochemical and histoarchitectural evaluation. Exp Toxicol Pathol 60: 397-404, 2008.

11. Priscilla DH and Prince PS: Cardioprotective effect of gallic acid on cardiac troponin-T, cardiac marker enzymes, lipid peroxidation products and antioxidants in experimentally induced myocardial infarction in Wistar rats. Chem Biol Interact 179: 118-124, 2009.

12. Lin CC and Shieh DE: The anti-inflammatory activity of Scutellaria rivularis extracts and its active components, baicalin, baicalein and wogonin. Am J Chin Med 24: 31-36, 1996.

13. Gabrielska J, Oszmiański J, Zyłka R and Komorowska M: Antioxidant activity of flavones from Scutellaria baicalensis in lecithin liposomes. Z Naturforsch C 52: 817-823, 1997.

14. Shao ZH, Vanden Hoek TL, Qin Y, et al: Baicalein attenuates oxidant stress in cardiomyocytes. Am J Physiol Heart Circ Physiol 282: H999-H1006, 2002.

15. Woo AY, Cheng $\mathrm{CH}$ and Waye MM: Baicalein protects rat cardiomyocytes from hypoxia/reoxygenation damage via a prooxidant mechanism. Cardiovasc Res 65: 244-253, 2005.

16. $\mathrm{Yu} \mathrm{W}, \mathrm{Liu} \mathrm{Q}$ and $\mathrm{Zhu} \mathrm{S}$ : Carvacrol protects against acute myocardial infarction of rats via anti-oxidative and anti-apoptotic pathways. Biol Pharm Bull 36: 579-584, 2013.

17. Kuo CP, Wen LL, Chen CM, et al: Attenuation of neurological injury with early baicalein treatment following subarachnoid hemorrhage in rats. J Neurosurg 199: 1028-1037, 2013.

18. Maslov LN, Lishmanov YB, Oeltgen PR, et al: Activation of peripheral delta2 opioid receptors increases cardiac tolerance to ischemia/reperfusion injury: Involvement of protein kinase C, NO-synthase, KATP channels and the autonomic nervous system. Life Sci 84: 657-663, 2009.

19. Papazzo A, Conlan X, Lexis L and Lewandowski P: The effect of short-term canola oil ingestion on oxidative stress in the vasculature of stroke-prone spontaneously hypertensive rats. Lipids Health Dis 10: 180, 2011.

20. Guo J, Li HZ, Wang LC, et al: Increased expression of calcium-sensing receptors in atherosclerosis confers hypersensitivity to acute myocardial infarction in rats. Mol Cell Biochem 366: 345-354, 2012.

21. Ming X, Tongshen W, Delin W and Ronghua Z: Cardioprotective effect of the compound yangshen granule in rat models with acute myocardial infarction. Evid Based Complement Alternat Med 2012: 717123, 2012.

22. Katus HA, Remppis A, Scheffold T, Diederich KW and Kuebler W: Intracellular compartmentation of cardiac troponin $\mathrm{T}$ and its release kinetics in patients with reperfused and nonreperfused myocardial infarction. Am J Cardiol 67: 1360-1367, 1991

23. Wolfrum S, Dendorfer A, Schutt M, et al: Simvastatin acutely reduces myocardial reperfusion injury in vivo by activating the phosphatidylinositide 3-kinase/Akt pathway. J Cardiovasc Pharmacol 44: 348-355, 2004.

24. Pipaliya $\mathrm{H}$ and Vaghasiya $\mathrm{J}$ : Cardio protective effect of vitamin A against isoproterenol-induced myocardial infarction. J Nutr Sci Vitaminol (Tokyo) 58: 402-407, 2012.

25. Chang WT, Li J, Vanden Hoek MS, et al: Baicalein preconditioning protects cardiomyocytes from ischemia-reperfusion injury via mitochondrial oxidant signaling. Am J Chin Med 41: 315-331, 2013. 